\title{
Mass-radius relations for massive white dwarf stars
}

\author{
L. G. Althaus ${ }^{1, \star}$, E. García-Berro ${ }^{1,2}$, J. Isern ${ }^{2,3}$, and A. H. Córsico ${ }^{4,5, \star}$ \\ 1 Departament de Física Aplicada, Universitat Politècnica de Catalunya, Av. del Canal Olímpic, s/n, 08860 Castelldefels, Spain \\ e-mail: [leandro;garcia]@fa.upc.es \\ 2 Institut d'Estudis Espacials de Catalunya, Ed. Nexus, c/Gran Capità 2, 08034 Barcelona, Spain \\ e-mail: isern@ieec.fcr.es \\ 3 Institut de Ciències de l'Espai, C.S.I.C., Campus UAB, Facultat de Ciències, Torre C-5, 08193 Bellaterra, Spain \\ ${ }^{4}$ Facultad de Ciencias Astronómicas y Geofísicas, Universidad Nacional de La Plata, Paseo del Bosque s/n, (1900) La Plata, \\ Argentina \\ e-mail: acorsico@fcaglp.unlp.edu.ar \\ 5 Instituto de Astrofísica La Plata, IALP, CONICET, Argentina
}

Received 4 March 2005 / Accepted 18 July 2005

\begin{abstract}
We present detailed theoretical mass-radius relations for massive white dwarf stars with oxygen-neon cores. This work is motivated by recent observational evidence about the existence of white dwarf stars with very high surface gravities. Our results are based on evolutionary calculations that take into account the chemical composition expected from the evolutionary history of massive white dwarf progenitors. We present theoretical mass-radius relations for stellar mass values ranging from 1.06 to $1.30 M_{\odot}$ with a step of $0.02 M_{\odot}$ and effective temperatures from $150000 \mathrm{~K}$ to $\approx 5000 \mathrm{~K}$. A novel aspect predicted by our calculations is that the mass-radius relation for the most massive white dwarfs exhibits a marked dependence on the neutrino luminosity. Extensive tabulations for massive white dwarfs, accessible from our web site, are presented as well.
\end{abstract}

Key words. stars: evolution - stars: white dwarfs - stars: fundamental parameters

\section{Introduction}

White dwarfs are the end-product of the evolution of low- and intermediate-mass stars. Thus, they preserve important clues about the formation and evolution of our Galaxy. This information can be retrieved by studying their observed mass, kinematic and luminosity distributions, provided that we have good structural and evolutionary models for the progenitors of white dwarfs and for the white dwarfs themselves. In particular, two fundamental tools to analyze the properties of the white dwarf population as a whole are the white dwarf luminosity function and the mass distribution of white dwarfs. The former has been consistently used to obtain estimates of the age of the Galactic disk (Winget et al. 1987; García-Berro et al. 1988; Hernanz et al. 1994; Richer et al. 2000) and the past history of the galactic star formation rate (Noh \& Scalo 1990; Díaz-Pinto et al. 1994). The latter yields important information about the late stages of stellar evolution since it reveals the amount of mass lost during stellar evolution from an initial stellar mass distribution (Liebert et al. 2005). Moreover, white dwarfs have been proposed to contribute significantly to the mass budget of our Galaxy - see García-Berro et al. (2004) for a recent discussion on this topic.

^ Member of the Carrera del Investigador Científico y Tecnológico, CONICET, Argentina.
However, the fundamental parameters of white dwarf stars, such as their individual masses and radii, are difficult to estimate. Hence, reliable mass and radius determinations are only available for a relatively small fraction of the whole local white dwarf population. Consequently, one of the most important theoretical results, namely the mass-radius relation, is poorly constrained by the observations, despite the improved evolutionary sequences available nowadays (Benvenuto \& Althaus 1999; Hansen 1999; Salaris et al. 2000).

A good determination of the white dwarf mass distribution provides useful constraints to the initial-final mass relationship, a key ingredient for modelling the different white dwarf populations (thin and thick disk, spheroid ...), and a touchstone for the stellar evolutionary models. Several works have focused on this issue. For instance, Finley et al. (1997) determined the effective temperatures and surface gravities of 174 DA white dwarfs with $T_{\text {eff }}>25000 \mathrm{~K}$, and from that they obtained the mass distribution of hot DA white dwarfs. Marsh et al. (1997) describe the results for a sample of 89 EUVselected white dwarfs and Vennes (1999) determined the mass distribution for a sample of 141 EUV-selected white dwarfs. Silvestri et al. (2001) studied the gravitational redshifts and mass distribution of 41 white dwarfs in common proper motion binary systems. Finally, Liebert et al. (2005) studied a complete sample of 348 DA white dwarfs from the Palomar Green survey. All these studies report the existence of a clear, well 
defined peak at $\sim 0.6 M_{\odot}$ with a typical dispersion of $\sim 0.1 M_{\odot}$. Additionally, a secondary peak is also reported at masses between 1.0 and $1.2 M_{\odot}$. These high-mass objects should have ONe cores, the primary products of the ${ }^{12} \mathrm{C}$ nuclear reactions, in contrast with average-mass white dwarfs, which undoubtedly have $\mathrm{CO}$ cores. The origin of these high-mass white dwarfs is uncertain, and it has been suggested that they could be the result of either binary evolution (Marsh et al. 1997) - but see Guerrero et al. (2004) - or of the evolution of heavy-weight intermediate-mass single stars (Ritossa et al. 1996; GarcíaBerro et al. 1997; Iben et al. 1997; Ritossa et al. 1999). In any case these white dwarfs do exist and a good mass-radius relation is needed in order to accurately determine their respective masses. All the mass determinations, including the most recent ones, rely on the evolutionary sequences of Wood (1995), which are valid for $\mathrm{CO}$ cores. To the best of our knowledge no reliable mass-radius relations for massive white dwarfs with ONe cores exist today and, thus, this is precisely the goal of the present work. Our paper is organized as follows. In Sect. 2 we present our input physics. In Sect. 3 we discuss our evolutionary sequences. Finally, in the last section we summarize our findings and draw our conclusions.

\section{Input physics}

The evolutionary code used in this paper is that used in our recent work about the evolution and pulsational properties of massive white dwarfs with oxygen and neon cores (Córsico et al. 2004), and we refer the reader to that paper for details about the input physics and about the procedure followed to obtain the initial white dwarf configurations. The code is based on updated and delailed constitutive physics. Briefly, the equation of state for the low-density regime includes an updated version of the equation of state of Magni \& Mazzitelli (1979). For the high-density regime, partially degenerate electrons, radiation pressure, ionic contributions and Coulomb interactions are considered. Radiative opacities are those of OPAL (Iglesias \& Rogers 1996) including carbon- and oxygen-rich compositions, complemented at low temperatures with the Alexander \& Ferguson (1994) molecular opacities. Neutrino emission rates for pair, photo, plasma and bremsstrahlung processes, and high-density conductive opacities are taken from Itoh et al. (1996a,b), and Itoh et al. (1994). Convection is treated in the framework of the mixing length theory as given by the ML2 parameterization (Tassoul et al. 1990). In this paper, we have neglected the effects of crystallization. Because we are interested in the calculation of mass radius relations, this assumption bears virtually no relevance. However, it is worth noting that crystallization in massive white dwarfs takes place at such high stellar luminosities that its impact on the white dwarf cooling is less relevant. In addition, chemical redistribution due to phase separation upon crystallizaton of the oxygen-neon core is of minor importance - see Córsico et al. (2004) for details and has not been considered in this work.

Our starting stellar configurations correspond to hot white dwarf structures with a realistic chemical stratification appropriate to white dwarfs resulting from progenitor stars of solar metallicity that are expected to have burnt carbon in semidegenerate conditions. All the evolutionary sequences considered in this work have the same chemical abundance profiles, and correspond to that illustrated in Fig. 4 of Córsico et al. (2004). Although minor changes in the chemical profile are expected because of the different masses of progenitor objects, these have a negligible influence in the mass-radius relation. The outer layer chemical stratification consists of a pure hydrogen envelope of $1.4 \times 10^{-6} M_{*}$ overlying a pure helium shell of $4 \times 10^{-4} M_{*}$ and, below that, a buffer rich in ${ }^{12} \mathrm{C}$ and ${ }^{16} \mathrm{O}$. We mention that the amount of hydrogen we adopted is an upper limit as imposed by nuclear reactions. We also find that the mass-radius relations are almost insensitive to the hydrogen envelope mass. The core is primarily composed of ${ }^{16} \mathrm{O}$ and ${ }^{20} \mathrm{Ne}$, plus some traces of ${ }^{12} \mathrm{C},{ }^{23} \mathrm{Na}$ and ${ }^{24} \mathrm{Mg}$. The chemical composition of the core is the result of repeated shell flashes that take place during the carbon burning phase in massive white dwarf progenitors (García-Berro et al. 1997). The oxygen-neon core of our models is characterized by flat profiles that are the result of a rehomogenization process induced by Rayleigh-Taylor instabilities in regions with negative molecular weight gradients, a process that is expected to take place along the white dwarf evolutionary sequence. We mention that the lowest stellar mass we considered in this study is close to the theoretical lower limit of $\approx 1.05 M_{\odot}$ for an oxygen-neon white dwarf to be formed (Gil-Pons et al. 2003).

The initial stellar models needed to start our cooling sequences were obtained by means of the same artificial evolutionary procedure described in Gautschy \& Althaus (2002) and Althaus \& Córsico (2004). Briefly, we start from a hot $0.95 M_{\odot}$ white dwarf model obtained self-consistently by evolving the progenitor star initially on the ZAMS (Althaus et al. 2003) to the white dwarf regime. In order to obtain initial white dwarf configurations for the different masses studied here we scaled the stellar mass and incorporated an artificial energy generation rate until the white dwarf reaches a luminosity far larger than that of the initial models considered here as meaningful. We checked the thermal structure of the models and found that the transitory state has negligible influence on the thermal configuration of the white dwarf interior well before the initial configuration is reached (see next section). Specifically, we checked the thermal structure of the $1.28 M_{\odot}$ model by extending the heating artificial procedure even further beyond the knee at high luminosities in the Hertzsprung-Russell diagram, as shown in Fig. 1. In this figure, the thick line displays the evolutionary track for one of our standard sequences (that of the $1.28 M_{\odot}$ model), whilst the thin line corresponds to the cooling track obtained when the artificial heating is extended to much larger luminosities. As can be seen the two evolutionary tracks are indistinguishable, thus confirming that the evolutionary results presented below do not depend on the initial conditions.

We have computed the evolution of massive white dwarf configurations from hot effective temperature stages down to very low luminosities. The values of the stellar mass considered range from 1.06 to $1.30 M_{\odot}$ with a step of $0.02 M_{\odot}$. The lower limit of this mass range corresponds to the minimum mass for a white dwarf to have an ONe core, whereas the upper limit corresponds to white dwarf masses for which post-newtonian corrections start to be important. Nevertheless, it is worth noting 


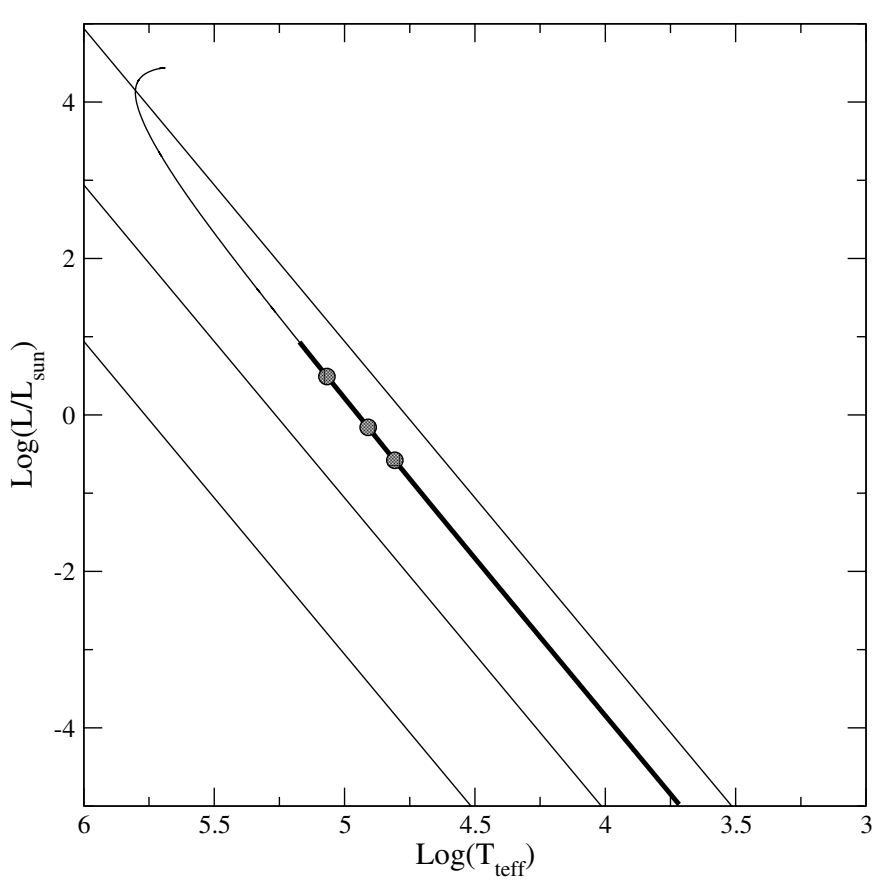

Fig. 1. Hertzsprung-Russell diagram for two $1.28 M_{\odot}$ evolutionary sequences with different artificial heating. Lines of constant radius for $R=0.01,0.001$ and $0.0001 R_{\odot}$ (from top to bottom) are also shown. See text for details.

that extremely massive white dwarfs - those with $M_{*} \gtrsim 1.3 M_{\odot}$ - are very unusual. The mass-radius relations are presented for effective temperature values ranging from about $150000 \mathrm{~K}$ to $\approx 5000 \mathrm{~K}$.

\section{Evolutionary results}

We begin by examining Fig. 2, which shows the internal temperature as a function of the outer mass fraction, $\log (1-$ $M_{\mathrm{r}} / M_{*}$ ), for three selected evolutionary stages of the $1.28 M_{\odot}$ white dwarf sequence corresponding, from top to bottom, to total luminosities of about $\log L / L_{\odot}=0.49,-0.16$ and -0.58 , respectively, which are marked as dots in Fig. 1. As can be seen the three models are already at advanced evolutionary stages, and, consequently, their thermal structure should be reliable. The top panel refers to our standard sequence, whilst the bottom panel displays the situation for models belonging to the sequence that started at very high luminosities (denoted by thin line in Fig. 1). Note that the temperature profiles of the models are the same for both sequences, reflecting the correctness of our artificial procedure to generate starting white dwarf configurations.

It is well known that neutrino losses considerably affect both the cooling time-scales and the structural properties of hot white dwarfs. Indeed, neutrino emission constitutes the most important physical process affecting the evolution of very hot $\mathrm{CO}$ white dwarfs, regardless of their mass. As can be seen in Figs. 2 and 3, this statement is also true for the massive ONe white dwarfs studied here. Note that, for the most massive model and for the luminosity range considered here, neutrino losses do not cause the maximum temperature to occur off-center (top panel of Fig. 2), as it is the case for the less

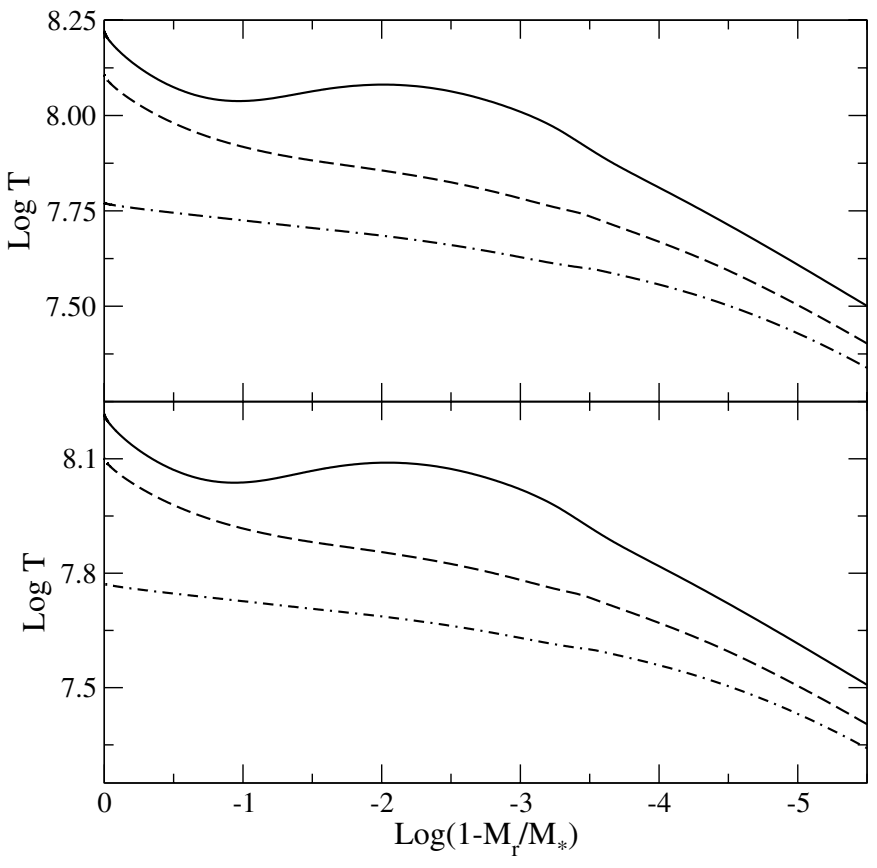

Fig. 2. Internal temperature in terms of the outer mass fraction. The top and bottom panel correspond, respectively, to three selected models from the evolutionary sequences depicted with thin and thick lines in Fig. 1. Solid, dashed and dot-dashed lines correspond to luminosities of about $\log L / L_{\odot}=0.49,-0.16$ and -0.58 , respectively. Note that for the two most luminous models, the internal temperature is strongly influenced by neutrino emission.

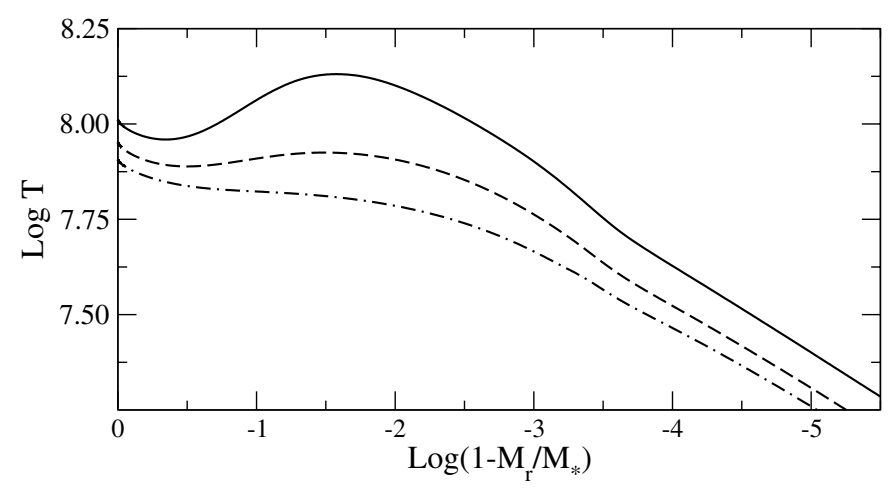

Fig. 3. Internal temperature in terms of the outer mass fraction. Solid, dashed and dot-dashed lines correspond to the $1.06 M_{\odot}$ white dwarf models at $\log L / L_{\odot}=0.66,-0.02$ and -0.38 , respectively. Note that for the most luminous model, the maximum temperature is off-center.

massive model of $1.06 M_{\odot}$, which is displayed in Fig. 3. In particular it is worth noting that for the $1.28 M_{\odot}$ white dwarf cooling sequence, the model with the largest photon luminosity - solid lines in Fig. 2 - temperature shows a local maximum at $\log \left(1-M_{\mathrm{r}} / M_{*}\right) \simeq-2.2$, well inside the ONe core. This can be understood by examining Fig. 4, which shows the neutrino emission rates (in erg/g/s) as a function of the mass fraction for the $1.28 M_{\odot}$ white dwarf model sequence. Note that the maximum neutrino emissivity occurs towards the outer edge of the degenerate core, with plasma neutrino being the dominant contribution. Indeed, for massive white dwarfs, the emission rate resulting from plasma neutrino is characterized by 


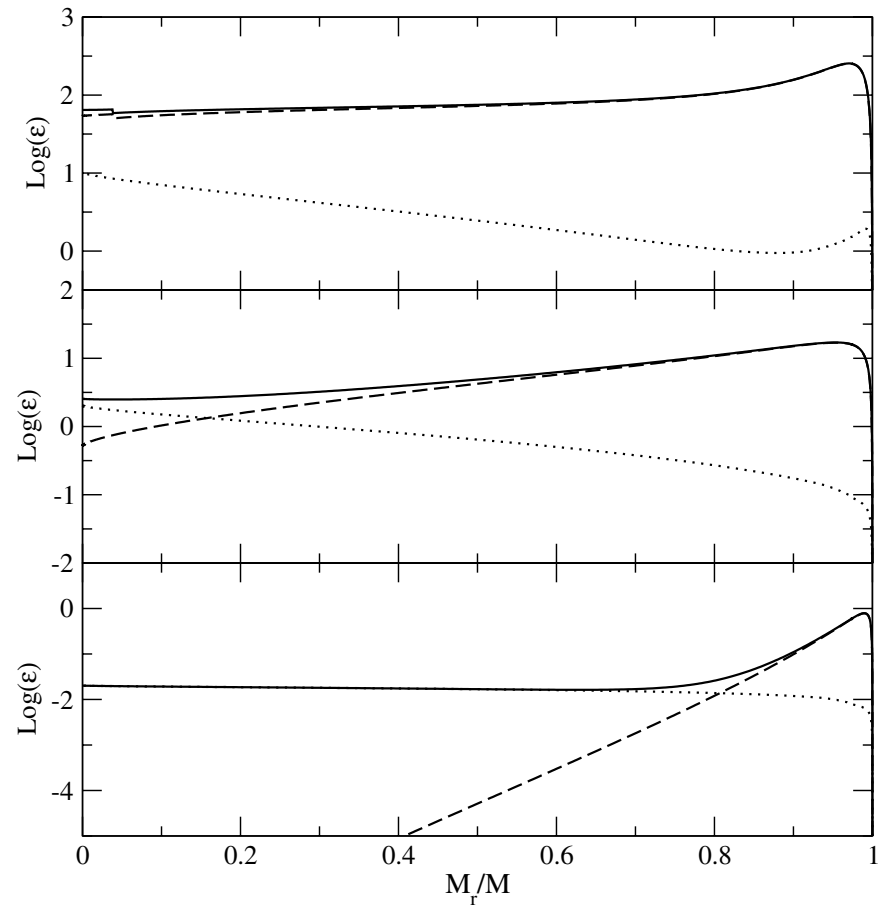

Fig. 4. Neutrino emission rate (in $\mathrm{erg} / \mathrm{g} / \mathrm{s}$ ) in terms of the mass fraction for three selected $1.28 M_{\odot}$ stellar models. Solid, dotted and dashed lines correspond to the total rate of neutrino emission, and to the rates due to brehmsstrahlung and plasma processes, respectively. The top, central and bottom panel correspond to luminosities of about $\log L / L_{\odot}=0.51,-0.17$ and -0.58 .

a maximum at densities much lower than the central densities of such models, as clearly shown, for instance, in Fig. 6 of Itoh et al. (1996b).

During the first evolutionary stages of the white dwarf models studied here - those with $\log L / L_{\odot} \gtrsim-0.75$ - most of the neutrino emission proceeds via the plasma process, as it is apparent from Fig. 5 (see also Fig. 4). However, for the most massive white dwarfs, neutrino losses through the bremsstrahlung process are not negligible whatsoever, being comparable to or even larger than the photon luminosity of the white dwarf. This is due to the strong dependence of the neutrino emissivity on the density and on the average charge of the ions. Moreover, the neutrino luminosity far exceeds the photon luminosity during these early stages of the evolution.

Neutrino losses constitute a powerful sink of energy in hot massive white dwarfs and leave their signatures in the massradius relations of the sequences, as it is apparent from Fig. 6. Indeed, as neutrino emission gradually decreases the models undergo an appreciable change in their radius. This effect is clearly noticeable in the $1.28 M_{\odot}$ white dwarf model shown in the bottom panel of Fig. 6, which shows a marked reduction in the radius of the model as the neutrino emission becomes extinct below $\log L / L_{\odot} \approx-0.25$. Note that this reduction in the stellar radius is absent if neutrino losses are not considered (the corresponding evolutionary sequence is represented by a dashed line in the botton panel of Fig. 6). This coupling, which is not found for stellar masses smaller than about $1.20 M_{\odot}$, has not been reported before. We stress that this behaviour is

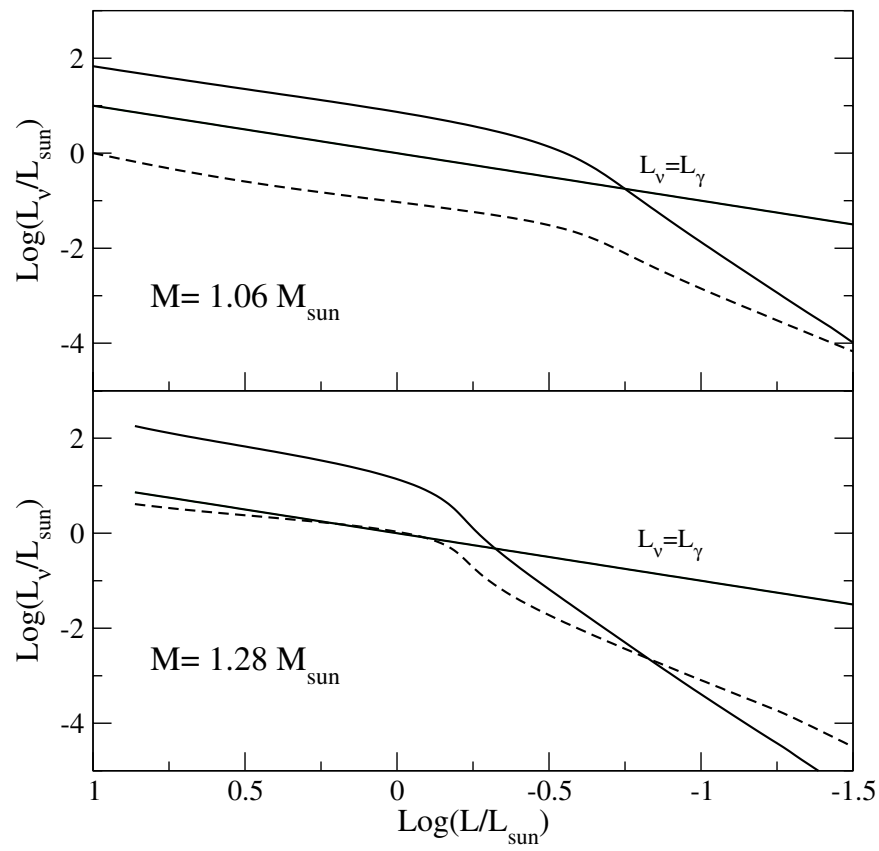

Fig. 5. Neutrino emission luminosity resulting from plasma and bremsstrahlung processes (solid and dashed lines, respectively) for the 1.06 and $1.28 M_{\odot}$ white dwarf sequences. In the interests of comparison, we depict the situation for which neutrino energy losses are equal to the photon luminosity of the white dwarf.

only noticeable for very massive white dwarf models, because in these models the maximum of neutrino emission occurs in the outer, less degenerate regions of the core. On the contrary, in less massive white dwarfs, neutrino energy losses are large in the degenerate core of the white dwarf. The upper panel of Fig. 6 illustrates the behaviour of neutrino luminosity, $L_{\gamma}$, as a function of photon luminosity. Here, the various neutrino processes are shown in the same plot. Note that neutrino energy losses in massive white dwarfs far exceed the photon luminosity. As the evolution proceeds, the neutrino luminosity fades away faster than the photon luminosity and neutrino cooling has little effect on the subsequent evolution of the models. This behaviour can be explained with the help of Fig. 6 as follows. As it has been already shown, for the most massive ONe white dwarfs the neutrino emissivity peaks in the outer partially degenerate layers of the white dwarf. Hence, these layers are cooler than in the case in which neutrino emission has been disregarded. Consequently, the radius of the white dwarf is smaller for the sequence in which neutrino emission has been properly taken into account. Since the peak of the neutrino emissivity is located at the edge of the degenerate core, in a region in which the temperature gradient is large, the nearly isothermal core provides enough energy to prevent the temperature to drop very rapidly in these regions. As a consequence the rate of change of the stellar radius is smaller than in the case in which neutrinos are disregarded. Moreover, since these layers are already cooler than those of the model in which neutrino emission is disregarded the rate of change of the radius is smaller as well. Nevertheless, in these layers neutrino cooling is efficient and, therefore, the temperature decreases and the density increases. This, ultimately leads to a decrease of the neutrino luminosity, 


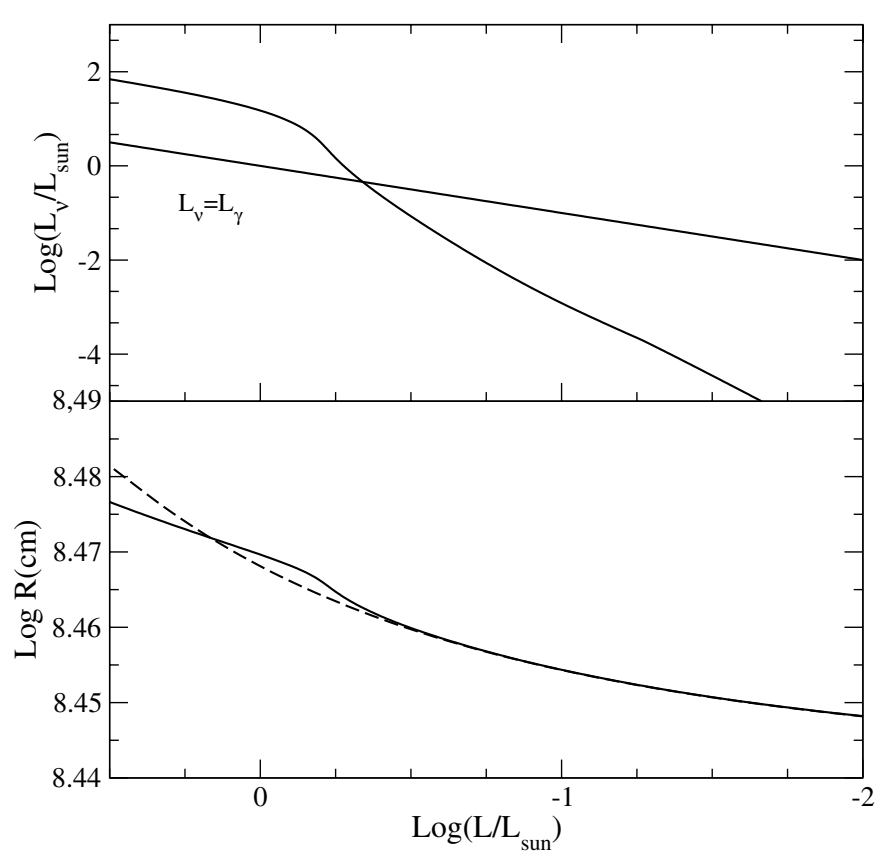

Fig. 6. Upper panel: evolution of the neutrino emission luminosity in terms of the stellar luminosity for the $1.28 M_{\odot}$ white dwarf sequence. Bottom panel: stellar radius as a function of stellar luminosity for the $1.28 M_{\odot}$ white dwarf sequence. The dashed line corresponds to the case in which neutrino losses have not been considered in the evolutionary sequence. Note the change in the stellar radius at $\log L / L_{\odot} \approx-0.25$ as neutrino losses fade away.

which finally drops below the photon luminosity. At this stage the radius of the evolutionary sequence in which neutrinos are taken into account is indistinguishable from that in which neutrinos has been disregarded.

The main result of our evolutionary calculations is summarized in Fig. 7, which shows the evolution of the surface gravity (in cgs units) for all our sequences as a function of the effective temperature. As it is well known, for a given effective temperature, more massive models are characterized by smaller radii and larger gravities. As cooling gradually proceeds, the surface gravity becomes larger, eventually reaching an almost constant value corresponding to that of the zero-temperature configuration, as it should be expected for strongly degenerate configurations. Note that finite-temperature effects are appreciable only at high effective temperatures, in the early evolutionary phases.

Because of the presence of abundant ${ }^{20} \mathrm{Ne}$ in the core of our massive white dwarf models, gravities are larger than those obtained when a $\mathrm{CO}$ core is adopted. This is a result of the non-ideal, corrective terms of the equation of state. In fact, the presence of ${ }^{20} \mathrm{Ne}$ yields a much stronger interacting plasma than that of a regular $\mathrm{CO}$ mixture adopted in the previous works (Wood 1995). This is borne out by Fig. 8 which illustrates the evolution of the surface gravity for selected massive white dwarf models with different core chemical composition. Specifically, solid, dashed and dot-dashed lines correspond to core compositions of oxygen-neon, oxygen and carbon, respectively. As expected, for a given stellar mass, surface gravities are larger the higher the atomic number $Z$ of the chemical constituent. Interestingly enough, very massive white dwarfs are

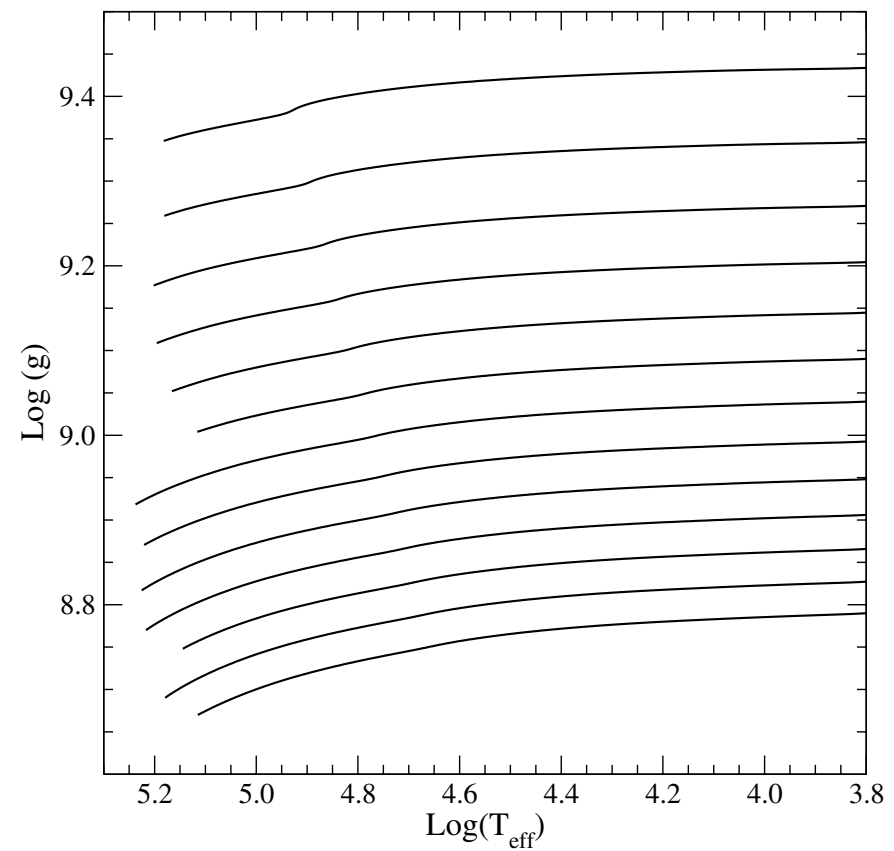

Fig. 7. Surface gravity as a function of effective temperature for all of the massive white dwarf evolutionary sequences considered in this work. From bottom to top, curves correspond to sequences with stellar masses from 1.06 to $1.30 M_{\odot}$ with a mass step of $0.02 M_{\odot}$. As neutrino emission becomes extinct at high effective temperatures, models becomes more compact, as it is reflected by the sudden change in the surface gravity, particularly for most massive models. For such sequences and advanced stages of evolution, finite-temperature effects are barely noticeable.

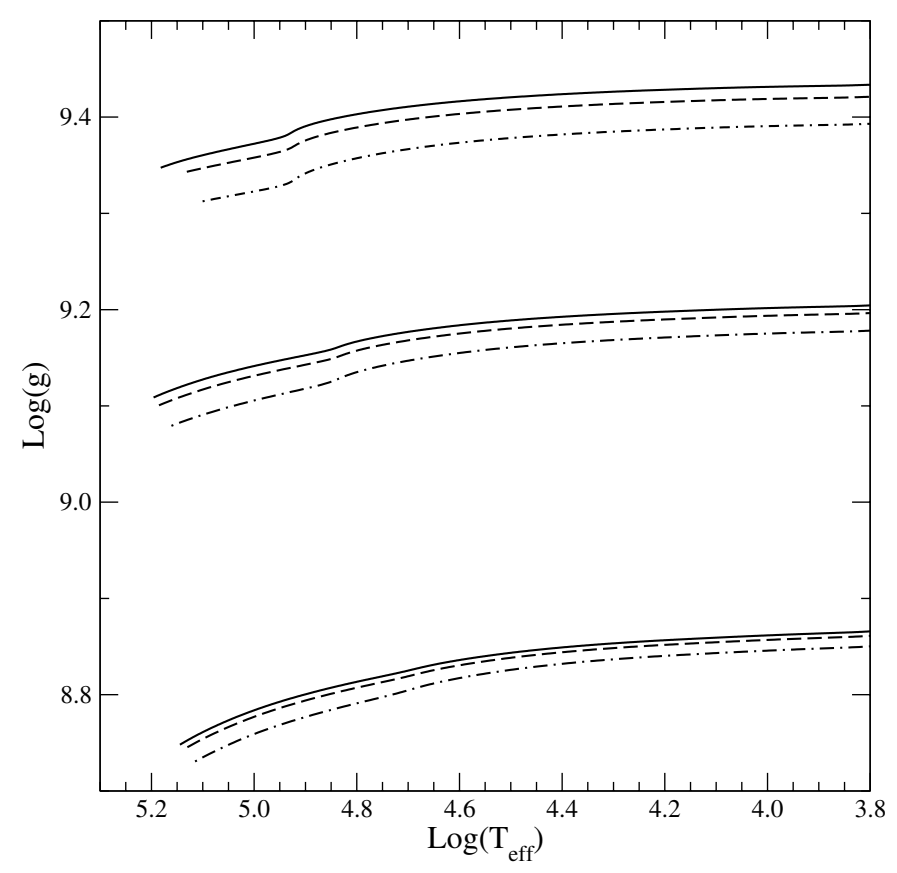

Fig. 8. Surface gravity as a function of effective temperature for the $1.30,1.24$ and $1.10 M_{\odot}$ white dwarf sequences with various core chemical compositions. Solid, dashed and dot-dashed lines correspond to core compositions of oxygen-neon, oxygen and carbon, respectively. Note the effect of the core chemical composition on surface gravity, particulary for very massive white dwarfs. 
Table 1. Surface gravities for white dwarf models with different core chemical composition at $T_{\text {eff }}=20000 \mathrm{~K}$.

\begin{tabular}{lll}
\hline \hline$M_{*} / M_{\odot}$ & $X_{\text {core }}$ & $\log (g)$ \\
\hline 1.10 & ONe & 8.853 \\
& O & 8.848 \\
& $\mathrm{C}$ & 8.837 \\
1.24 & ONe & 9.195 \\
& O & 9.187 \\
& $\mathrm{C}$ & 9.168 \\
1.30 & & \\
& ONe & 9.426 \\
& O & 9.414 \\
& $\mathrm{C}$ & 9.385 \\
\hline
\end{tabular}

rather sensitive to the adopted core chemical composition. Such white dwarfs are characterized by densities high enough for electrons to be extremely relativistic throughout most of their interiors. Hence, Coulomb interactions grow in importance as the stellar mass increases. Finally, in Table 1 we list the surface gravity values expected at $T_{\text {eff }}=20000 \mathrm{~K}$ for some selected white dwarf sequences with different core chemical composition.

\section{Conclusions}

We have computed reliable mass-radius relations - or, equivalently, $\log g / T_{\text {eff }}$ relations - for massive white dwarfs with degenerate $\mathrm{ONe}$ cores. Our calculations encompass masses ranging from 1.06 to $1.30 M_{\odot}$, the expected range of masses for which $\mathrm{ONe}$ white dwarfs should presumably exist. To the best of our knowledge this is the first attemtpt to compute such a relation with a realistic equation of state - which includes the non-ideal, corrective terms and the full temperature dependence - and reliable chemical profiles for the degenerate interior reflecting the previous evolutionary history of the progenitors of such white dwarfs. The results presented here will undoubtedly be helpful in the interpretation of recent observations of white dwarf stars with very high surface gravities (Dahn et al. 2004; Madej et al. 2004; Nalezyty \& Madej 2004), which up to now relies on prior evolutionary sequences which were computed assuming CO cores. Moreover, we have shown that the mass-radius relation shows a marked dependence on the neutrino luminosity, in such a way that for the most massive stars within this mass range a sudden decrease in the massradius relationship shows up shortly before the neutrino luminosity drops below the photon luminosity.

Finally, we have prepared detailed tabulations of radius and gravity for all our massive white dwarf sequences, which are available at our web site:

http://www . fcaglp. unlp.edu. ar/evolgroup/.
Acknowledgements. This research was partially supported by the Instituto de Astrofísica La Plata, by the MCYT grant AYA04094C03-01 and 02, by the European Union FEDER funds, and by the CIRIT. L.G.A. acknowledges the Spanish MCYT for a Ramón y Cajal Fellowship.

\section{References}

Alexander, D. R., \& Ferguson, J. W. 1994, ApJ, 437, 879

Althaus, L. G., \& Córsico, A. H. 2004, A\&A, 417, 1115

Althaus, L. G., Serenelli, A. M., Córsico, A. H., \& Montgomery, M. H. 2003, A\&A, 404, 593

Benvenuto, O. G., \& Althaus, L. G. 1999, MNRAS, 303, 30

Córsico, A. H., García-Berro, E., Althaus, L. G., \& Isern, J. 2004, A\&A, 427, 923

Dahn, C. C., Bergeron, P., Liebert, J., et al. 2004, ApJ, 605, 400

Díaz-Pinto, A., García-Berro, E., Hernanz, M., Isern, J., \& Mochkovitch, R. 1994, A\&A, 282, 86

Finley, D. S., Koester, D., \& Basri, G. 1997, ApJ, 488, 375

García-Berro, E., Hernanz, M., Isern, J., \& Mochkovitch, R. 1988, Nature, 333, 642

García-Berro, E., Ritossa, C., \& Iben, I. 1997, ApJ, 485, 765

García-Berro, E., Torres, S., Isern, J., \& Burkert, A. 2004, A\&A, 418, 53

Gautschy, A., \& Althaus, L. G. 2002, A\&A, 382, 141

Gil-Pons, P., García-Berro, E., José, J., Hernanz, M., \& Truran, J. W. 2003, A\&A, 407, 1021

Guerrero, J., García-Berro, E. \& Isern, J. 2004, A\&A, 413, 257

Itoh, N., Hayashi, H., \& Kohyama, Y. 1994, ApJ, 416, 438

Itoh, N., Nishikawa, A., \& Kohyama, Y. 1996a, ApJ, 470, 101

Itoh, N., Hayashi, H., Nishikawa, A., \& Kohyama, Y. 1996b, ApJS, 102,41

Hansen, B. 1999, ApJ, 520, 680

Hernanz, M., García-Berro, E., Isern, J., et al. 1994, ApJ, 434, 652

Iben, I., Ritossa, C., \& García-Berro, E. 1997, ApJ, 489, 772

Iglesias, C. A., \& Rogers, F. 1996, ApJ, 464, 943

Liebert, J., Bergeron, P., \& Holberg, J. 2005, ApJS, 156, 47

Madej, J., Nalezyty, M., \& Althaus, L. G. 2004, A\&A, 419, L5

Magni, G., \& Mazzitelli, I. 1979, A\&A, 72, 134

Marsh, M. C., Barstow, M. A., Buckley, D. A., et al. 1997, MNRAS, 287, 705

Nalezyty, M., \& Madej, J. 2004, A\&A, 420, 507

Noh, H. R., \& Scalo, J. 1990, ApJ, 352, 605

Richer, H. B., Hansen, B., Limongi, M., et al. 2000, ApJ, 529, 318

Ritossa, C., García-Berro, E., \& Iben, I. 1996, ApJ, 460, 489

Ritossa, C., Garía-Berro, E., \& Iben, I. 1999, ApJ, 515, 381

Salaris, M., García-Berro, E., Hernanz, M., Isern, J., \& Saumon, D. 2000, ApJ, 544, 1036

Silvestri, N. M., Oswalt, T. D., Wood, M. A., et al. 2001, AJ, 121, 503

Tassoul, M., Fontaine, G., \& Winget, D. E. 1990, ApJS, 72, 335

Vennes, S. 1999, ApJ, 525, 995

Winget, D., Hansen, C., Liebert, J., et al. 1987 ApJ, 315, L77

Wood, M. A. 1995, in Proc. of the 9th European Workshop on White Dwarfs, ed. D. Koester, \& K. Werner (Berlin: Springer), 41 